\title{
Nanoscale Fourier-Transform Magnetic Resonance Imaging
}

\author{
John M. Nichol, ${ }^{1}$ Tyler R. Naibert, ${ }^{1}$ Eric R. Hemesath, ${ }^{2}$ Lincoln J. Lauhon, ${ }^{2}$ and Raffi Budakian ${ }^{1, *}$ \\ ${ }^{1}$ Department of Physics, University of Illinois at Urbana-Champaign, Urbana, Illinois 61801, USA \\ ${ }^{2}$ Department of Materials Science and Engineering, Northwestern University, Evanston, Illinois 60208, USA \\ (Received 15 February 2013; revised manuscript received 23 May 2013; published 26 September 2013; \\ publisher error corrected 7 October 2013)
}

\begin{abstract}
We report a method for nanometer-scale pulsed nuclear magnetic resonance imaging and spectroscopy. Periodic radio-frequency pulses are used to create temporal correlations in the statistical polarization of a solid organic sample. The spin density is spatially encoded by applying a series of intense magnetic field gradient pulses generated by focusing electric current through a nanometer-scale metal constriction. We demonstrate this technique using a silicon nanowire mechanical oscillator as a magnetic resonance sensor to image ${ }^{1} \mathrm{H}$ spins in a polystyrene sample. We obtain a two-dimensional projection of the sample proton density with approximately $10-\mathrm{nm}$ resolution.
\end{abstract}

DOI: 10.1103/PhysRevX.3.031016

Subject Areas: Magnetism, Nanophysics

\section{INTRODUCTION}

Nuclear magnetic resonance imaging (MRI) is a powerful technique for noninvasive three-dimensional biological and materials imaging [1]. In large part, MRI has proved so successful because it offers a host of sophisticated methods that can be used to image samples in a variety of informative ways. In general, all MRI techniques rely on accurate determination of magnetic resonance frequencies. For example, the locations of nuclear spins in a sample can be determined by applying an external magnetic field gradient, causing nuclear magnetic resonance (NMR) frequencies to vary in space. Nuclear spins of different types in the same sample can be distinguished because NMR frequencies are chemically specific. Even nuclear spins of the same type in different chemical environments can be distinguished (e.g., protons in fat and protons in water) via slight changes in resonance frequency as a result of the chemical shift interaction [2]. MRI can also probe local fluctuating magnetic fields through spin-relaxation weighted imaging [2]. Other well-known spectroscopic MRI techniques include functional magnetic resonance imaging [3], diffusion tensor imaging [4], and tomographic reconstruction [2], to name a few.

Each of these common MRI techniques, and indeed most modern NMR spectroscopic techniques, use radio-frequency (rf) pulses to generate a component of the sample magnetization perpendicular to the external magnetic field. The coherent precession of the magnetization is measured and Fourier transformed to yield the sample NMR spectrum. Since its discovery in 1966, pulsed Fourier-transform magnetic resonance [5] has revolutionized both NMR

\footnotetext{
*budakian@illinois.edu
}

Published by the American Physical Society under the terms of the Creative Commons Attribution 3.0 License. Further distribution of this work must maintain attribution to the author(s) and the published article's title, journal citation, and DOI. spectroscopy and MRI because it offers dramatically enhanced sensitivity over continuous-wave methods by allowing the simultaneous measurement of all spectral components [6]. In acquisition schemes such as Fourier [7,8] or Hadamard encoding [9], all components of the sample spectrum are averaged for the entire acquisition period. When detector noise is the limiting factor, these techniques significantly increase the signal-to-noise ratio (SNR) in what is known as the multiplex advantage over methods that acquire each element of the spectrum sequentially.

Because the nuclear magnetic moment is relatively weak [1], however, the spatial resolution of inductive MRI remains limited to millimeter length scales in common practice and a few micrometers in the highest-resolution experimental instruments [10]. Nonetheless, there is considerable interest in extending the resolution and sensitivity of magnetic resonance detection to enable spectroscopy and imaging on the nanometer scale. Promising work in this direction includes force-detected magnetic resonance [11], which has been used to perform three-dimensional imaging of single tobacco mosaic virus particles with resolution below $10 \mathrm{~nm}$ [12], and nitrogen-vacancy-based magnetic resonance $[13,14]$, which has been used to detect proton resonance in nanometer-sized volumes $[15,16]$.

In spite of this remarkable progress, the application of classic pulsed magnetic resonance techniques to nanoscale systems remains challenging because of two primary reasons. First, achieving high spatial resolution in nanoscale MRI generally requires intense static magnetic field gradients [12,17]. However, the presence of large static gradients makes uniform spin manipulation using $\mathrm{rf}$ pulses difficult and complicates NMR spectra. Second, pulsed magnetic resonance techniques cannot be used per se because statistical spin fluctuations exceed the Boltzmann spin polarization in nanoscale samples $[18,19]$. When the statistical polarization dominates, the projection of the sample magnetization along any axis fluctuates randomly in time. For objects at the micrometer scale and above, where the 
Boltzmann polarization dominates, many pulsed magnetic resonance techniques have been proposed [20] and demonstrated [21-25] in force-detected experiments.

In the following, we present a new paradigm in forcedetected magnetic resonance that overcomes both challenges to enable pulsed nuclear magnetic resonance in nanometer-size statistically polarized samples. In this proof-of-concept work, we demonstrate Fourier-transform spectroscopy and imaging with nanoscale resolution by periodically applying $\mathrm{rf}$ pulses to create correlations in the statistical polarization, or spin noise, of a solid organic sample. Gradient pulses for imaging are generated using ultrahigh current densities in a nanoscale metal constriction, and the spin-noise correlations are recorded for a set of pulse configurations and Fourier transformed to give the spin density. A silicon nanowire oscillator is used as a magnetic resonance sensor to reconstruct a two-dimensional projection image of the proton density in a polystyrene sample with roughly $10-\mathrm{nm}$ resolution. We also show that Fourier-transform imaging enhances sensitivity via the multiplex advantage for high-resolution imaging of statistically polarized samples. Most importantly, our protocol establishes a method by which all other pulsed magnetic resonance techniques can be used for nanoscale imaging and spectroscopy.

\section{APPARATUS}

Figure 1(a) shows a schematic of the apparatus. A key element of the experiment is an ultrasensitive silicon nanowire force transducer [26], which acts as the magnetic resonance sensor. The nanowire vibrates in response to the force of interaction between the protons in the sample and the time-varying inhomogeneous magnetic field produced by a nanometer-size constriction in a currentcarrying metal wire. The sample consists of a thin polystyrene coating on the tip of the silicon nanowire [Fig. 1(b)]. The nanowire used in this study is grown epitaxially on a $\mathrm{Si}[111]$ substrate using a controlled-diameter vapor-liquidsolid approach with silane as a precursor at $600^{\circ} \mathrm{C}$ [27]. The nanowire is roughly $15 \mu \mathrm{m}$ long, with a tip diameter of $50 \mathrm{~nm}$. The fundamental flexural mode has a spring constant $k=150 \mu \mathrm{N} / \mathrm{m}$, a resonance frequency $\omega_{0} / 2 \pi=$ $333 \mathrm{kH} z$, and an intrinsic quality factor $Q=1.8 \times 10^{4}$ at a temperature of approximately $6 \mathrm{~K}$. The displacement of the nanowire is measured using a polarized fiber-optic interferometer [26,28].

The current-carrying wire consists of a lithographically patterned constriction in a Ag film [Fig. 1(c)]. The constriction focuses current passing through the film to densities exceeding $3 \times 10^{8} \mathrm{~A} \mathrm{~cm}^{-2}$. Such locally intense current densities generate (1) large time-dependent magnetic field gradients that couple nuclear spins in the sample to the resonant displacement of the nanowire, (2) rf magnetic fields to excite magnetic resonance in the sample, and (3) pulsed gradients for imaging. The constriction used in
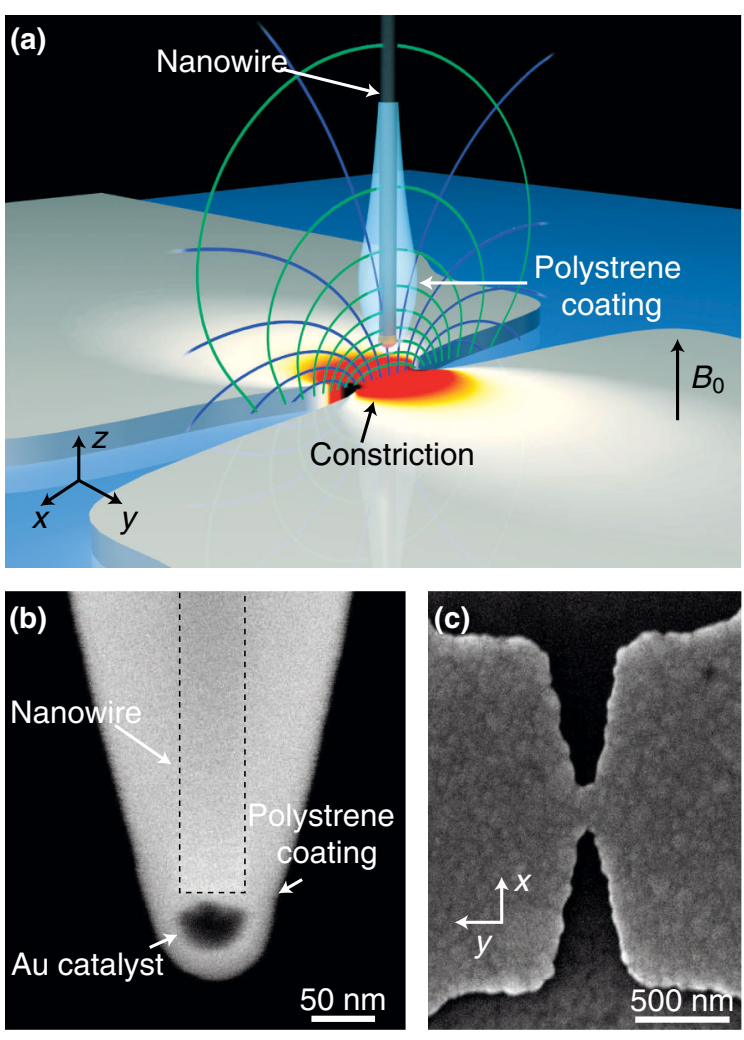

FIG. 1. Experimental apparatus. (a) Schematic of the experimental setup. A silicon nanowire coated with polystyrene is positioned near the constriction in a Ag current-carrying wire. The locally high current density through the constriction generates intense fields and gradients used for readout, spin manipulation, and spatial encoding. During imaging, the spin density is encoded along contours of constant Larmor and Rabi frequencies, which are illustrated as blue and green lines, respectively. (b) Scanning electron micrograph of a representative nanowire and polystyrene coating prepared in the same manner as the nanowire and sample used in this study. The actual nanowire and sample used here were not imaged in order to avoid electron damage. The dashed lines indicate the outer diameter of the nanowire. (c) Scanning electron micrograph of the constriction used in this study. The constriction, which appears in light gray in the image, is $100 \mathrm{~nm}$ thick and $240 \mathrm{~nm}$ wide.

this study is $240 \mathrm{~nm}$ wide and $100 \mathrm{~nm}$ thick (see the Supplemental Material [29]). Both the nanowire substrate and constriction are cooled to $4.2 \mathrm{~K}$ in high vacuum, and the sample is positioned $40 \mathrm{~nm}$ above the center of the constriction. A small superconducting solenoid provides the static field $B_{0}=0.183 \mathrm{~T}$ along the $z$ direction. We use the MAGGIC (modulated alternating gradients generated with currents) spin detection protocol [28] to measure the longitudinal component of the proton statistical polarization in the sample near the constriction. In the MAGGIC protocol, an oscillating electric current through the constriction generates a magnetic field gradient that alternates at the nanowire mechanical resonance frequency. The force of interaction between the spins in the sample and the 
alternating inhomogeneous magnetic field induces an angstrom-scale vibration of the nanowire, which is measured using the optical interferometer.

\section{SPIN-NOISE ENCODING}

Fourier-transform imaging and spectroscopy involve measuring the transverse component of the sample magnetization as it precesses around an external magnetic field after an rf excitation pulse. To detect the coherent precession of the magnetization using the MAGGIC protocol, we use an encoding pulse sequence that is related to a Ramsey-fringe measurement [30], which projects the coherent evolution of the magnetization onto the longitudinal axis. The sequence consists of an adiabatic half-passage (AHP) [31], an evolution period $t_{e}$, and a time-reversed AHP [Fig. 2(a)]. The first AHP rotates the spins away from the $z$ axis onto the $x y$ plane. During the period $t_{e}$, the spins precess about $B_{0}$. The second AHP, which is phase shifted by $\phi\left(t_{e}\right)=-\gamma B_{0} t_{e}$ relative to the first AHP, projects the magnetization back onto the $z$ axis [Fig. 2(b)]. Here, $\gamma / 2 \pi=42.6 \mathrm{MHz} / \mathrm{T}$ is the proton gryomagnetic ratio. The time-dependent phase shift creates a longitudinal projection that oscillates at the Larmor frequency as $t_{e}$ varies.

Because the statistical polarization fluctuates randomly, the encoding has no effect on the mean or variance of the polarization. If, however, the sequence is inserted repeatedly (e.g., every $\tau_{p}$, where $1 / \tau_{p}$ is the repetition rate) in the MAGGIC protocol, the encoding creates measurable correlations in the force signal, provided that $\tau_{p} \ll \tau_{m}$, where $\tau_{m}$ is the statistical spin-correlation time. In the Supplemental Material [29], we show that the time-averaged autocorrelation $\bar{R}_{f f}\left(\tau_{p}, t_{e}\right)$ of the force signal at lag $\tau_{p}$ is

$\bar{R}_{f f}\left(\tau_{p}, t_{e}\right)=\frac{e^{-\tau_{p} / \tau_{m}} \mu^{2} D^{2}}{2} \int d \boldsymbol{r} \rho(\boldsymbol{r}) G^{2}(\boldsymbol{r}) M\left(t_{e}, \boldsymbol{r}\right)$.

(a)

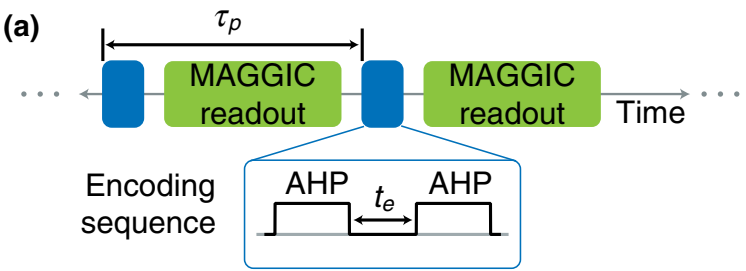

(b)
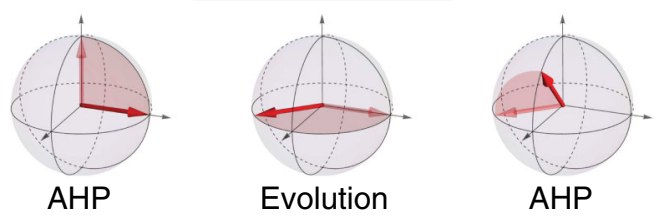

FIG. 2. Spin-noise encoding. (a) Periodic encoding pulses are inserted in the MAGGIC protocol every $\tau_{p}$. For the freeprecession measurement, the sequence consists of two AHPs separated by an evolution period $t_{e}$. (b) Illustration of the pulse sequence showing the evolution of a spin initially oriented along the $z$ axis.
Here, $\mu$ is the spin magnetic moment, $D$ is the MAGGIC gradient modulation duty cycle [28], $\rho(\boldsymbol{r})$ is the spin density, $G(\boldsymbol{r})$ is the gradient modulation strength, and $M\left(t_{e}, \boldsymbol{r}\right)$ describes the effect of the encoding. In particular, $M\left(t_{e}, r\right)=$ $1-2 P_{\text {flip }}\left(t_{e}, \boldsymbol{r}\right)$, where $P_{\text {flip }}\left(t_{e}, \boldsymbol{r}\right)$ is the probability for a spin located at $\boldsymbol{r}$ to reverse its orientation after a single encoding sequence. For example, if a single encoding pulse has a unit probability to invert the spin, then $M\left(t_{e}, \boldsymbol{r}\right)=-1$; if the encoding pulse has no effect on the spin orientation, $M\left(t_{e}, \boldsymbol{r}\right)=1$. The method presented here of measuring correlations in the spin noise is related to previous spectroscopic approaches [19,32-34] that correlate the polarization before and after an encoding pulse.

For the encoding sequence described above, $M\left(t_{e}, \boldsymbol{r}\right)=$ $E_{v}\left(t_{e}\right) \cos \left(\gamma B_{0} t_{e}\right)$. The free precession will decay with an envelope $E_{v}\left(t_{e}\right)$ because of the fluctuating local fields experienced by the spins. To verify the encoding procedure, we measure the Larmor precession of the statistical polarization by sweeping $t_{e}$ [Fig. 3(a)]. The decay envelope is reasonably well described by a Gaussian: $E_{v}\left(t_{e}\right)=e^{-\left(t_{e} / T_{2}^{*}\right)^{2}}$, with $T_{2}^{*}=14 \mu$ s [Fig. 3(b)], consistent with previous measurements in polystyrene [35]. This relatively short spin-dephasing time is caused by
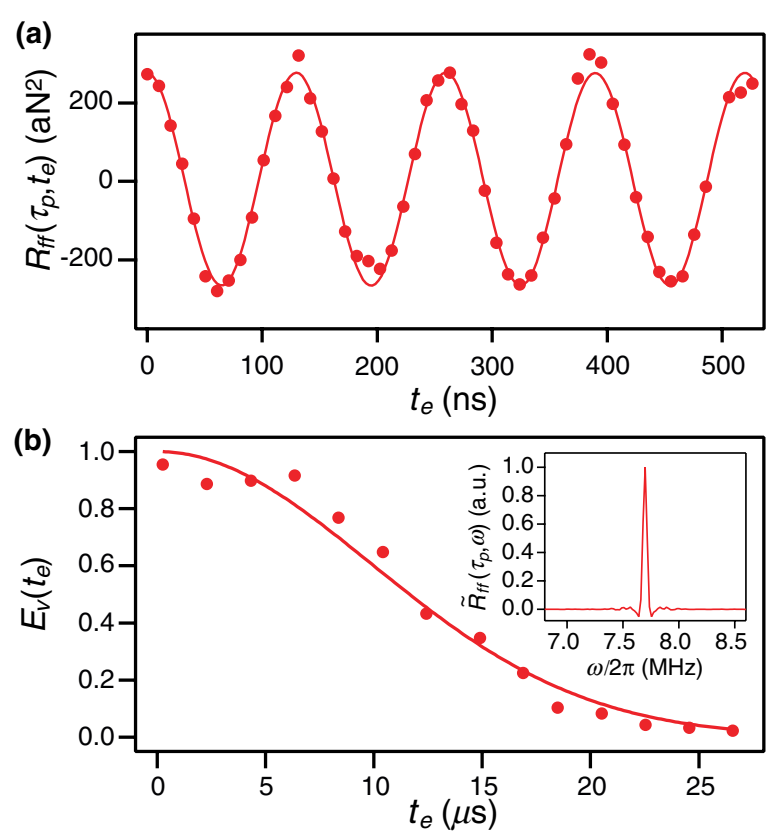

FIG. 3. Free precession in a statistically polarized sample. (a) Autocorrelation of the force signal $R_{f f}\left(\tau_{p}, t_{e}\right)$ and fit to a cosine. (b) Amplitude of the free precession and fit to a Gaussian. From the fit, we infer that $T_{2}^{*}=14 \mu \mathrm{s}$. Inset: Proton NMR spectrum of the statistically polarized sample. To obtain the spectrum, the data from (b) are linearly interpolated between points to give the precession envelope. The envelope is multiplied by a cosine at the measured precession frequency and cosine transformed to yield the spectrum. We attribute nonGaussian features in the spectrum to deviations in the time record from ideal Gaussian behavior, especially at short times. 
anisotropic dipole-dipole interactions in the solid polystyrene sample [36]. By cosine transforming the data, we obtain the nuclear magnetic resonance spectrum of the statistically polarized sample [Fig. 3(b)].

\section{FOURIER-TRANSFORM IMAGING}

The essential feature of the free-precession encoding is the use of repeated, identical pulse sequences to induce correlations in the spin noise. Such a paradigm permits the use of established pulsed magnetic resonance techniques not only for spectroscopy but also for imaging of statistically polarized samples. Fourier encoding, for example, uses a pulsed gradient during the free precession to encode the location of a spin in the phase or frequency of its Larmor precession. With the use of the constriction, which enables the generation of pulsed gradients, this technique can be adapted for nanoscale imaging.

Static current through the constriction produces a strong gradient in the $x$ direction of the total field $B_{\text {tot }}(\boldsymbol{r})$. Additionally, rf current through the constriction at frequency $\gamma B_{0}$ produces a field in the rotating frame $B_{1}(\boldsymbol{r})=$ $B_{x}(\boldsymbol{r}) / 2$, which varies strongly in the $z$ direction. These two independent gradients enable spatial encoding in two dimensions [Fig. 1(a)]. To simplify notation, we set $u \equiv$ $\omega_{\text {Rabi }}(\boldsymbol{r})=\gamma B_{1}(\boldsymbol{r})$ and $v \equiv \omega_{\text {Larmor }}(\boldsymbol{r})=\gamma B_{0}(\boldsymbol{r})$. Because neither $u$ nor $v$ varies appreciably with respect to $y$ over the dimensions of the sample for fixed $x$ and $z$, we make the reasonable assumptions that both $u$ and $v$ are independent of $y$ for the purposes of imaging, i.e., $u(r)=u(x, z)$ and $v(r)=v(x, z)$.

To encode the spin density along $v$ contours, a pulse sequence similar to the free-precession sequence is used, except that a static gradient pulse of length $t_{v}$ is applied during the evolution period [Fig. 4(a)]. To encode along $u$ contours, an rf pulse of length $t_{u}$ with center frequency $\gamma B_{0}$ is used to nutate spins about the effective field in the rotating frame by an angle $\gamma B_{1}(\boldsymbol{r}) t_{u}$. By incrementing the gradient pulse lengths, we record the Fourier transform of the two-dimensional projection of the spin density.

For the sequence discussed above, $M\left(t_{u}, t_{v}, \boldsymbol{r}\right)=$ $E_{u}\left(t_{u}\right) E_{v}\left(t_{v}\right) \cos \left[u(\boldsymbol{r}) t_{u}\right] \cos \left[v(\boldsymbol{r}) t_{v}\right]$, where $E_{u}\left(t_{u}\right)$ describes the transverse spin relaxation in the rotating frame [37]. Hence,

$$
\begin{aligned}
\bar{R}_{f f}\left(\tau_{p}, t_{u}, t_{v}\right)= & \frac{e^{-\tau_{p} / \tau_{m}} E_{u}\left(t_{u}\right) E_{v}\left(t_{v}\right) \mu^{2} D^{2}}{2} \\
& \times \int d u d v p(u, v) \cos \left(u t_{u}\right) \cos \left(v t_{v}\right),
\end{aligned}
$$

where $p(u, v)=G^{2}(u, v) J(u, v) \int d y \rho(y, u, v)$ is the projected signal density in the $(u, v)$ coordinate system, and $J(u, v)$ is the Jacobian of the $(x, z) \rightarrow(u, v)$ coordinate transformation. We also assume that the gradient is independent of $y$ for fixed $x$ and $z$ in the sample, i.e., $G(\boldsymbol{r})=$ $d B_{z}(x, z) / d x$.
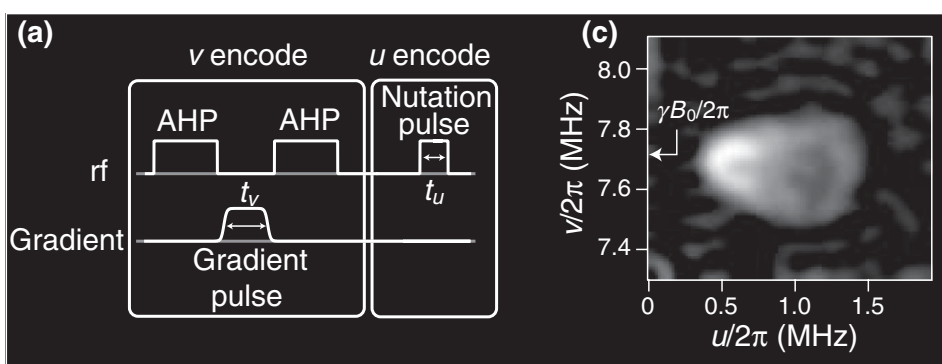

(d)

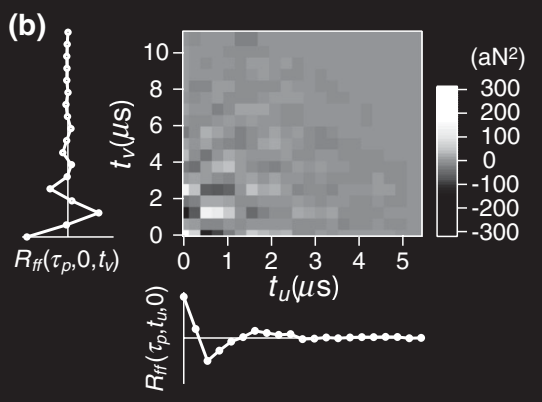

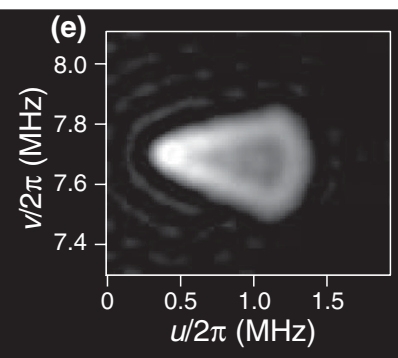

(f)

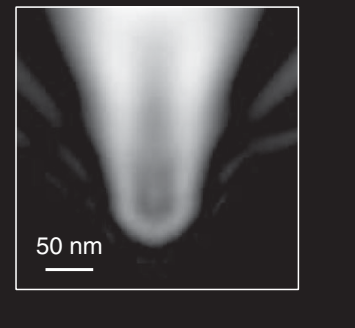

FIG. 4. Two-dimensional MRI of the polystyrene sample. (a) Image encoding sequence. In the $v$-encoding step, the spins precess in the presence of a gradient for a time $t_{v}$. In the $u$-encoding step, the spins precess about the effective field in the rotating frame for a time $t_{u}$. (b) Raw data. Cross sections corresponding to $R_{f f}\left(\tau_{p}, 0, t_{v}\right)$ and $R_{f f}\left(\tau_{p}, t_{u}, 0\right)$ are shown. (c) Signal density in the $(u, v)$ coordinate system obtained by cosine transforming the raw data. The arrow indicates the position of $\gamma B_{0} / 2 \pi$. (d) Real-space reconstruction of the projected spin density. The nanowire and gold catalyst are clearly visible through the polystyrene in the image as a reduction in the spin density. The cross sections above and to the right of the image are taken along the lines indicated by the arrows. (e) Simulated signal density in ( $u, v$ ) space calculated for the sample and nanowire geometry shown in Fig. 1(b). (f) Real-space reconstruction of the simulation in (e). 
To record an image, $R_{f f}\left(\tau_{p}, t_{u}, t_{v}\right)$ is measured for 305 different $\left(t_{u}, t_{v}\right)$ configurations [Fig. 4(b)]. The data are cosine transformed to obtain the frequency-space projection of the proton density [Fig. 4(c)] and the real-space representation of the proton density [Fig. 4(d)] (see the Supplemental Material [29]). The reconstructed spin density strongly resembles the expected shape of the polystyrene coating [Fig. 1(b)]. The nanowire and the gold catalyst particle are clearly visible through the polystyrene in the image as a reduction in the spin density. Figures 4(e) and 4(f) are simulations based on the expected shape of the sample [Fig. 1(b)]. Both simulations appear qualitatively similar to the actual data and image.

To determine the spatial resolution in the image, we simulate the image from a point source located near the tip of the sample and find the resolution in the $x$ and $z$ directions to be approximately 10 and $15 \mathrm{~nm}$, respectively. The spatial resolution is best at the tip of the sample where the gradients from the constriction are the largest (see the Supplemental Material [29]). The maximum imaging gradients in this study are $2.0 \times 10^{5} \mathrm{Tm}^{-1}$ in $z$ for $56 \mathrm{~mA}$ of current through the constriction and $1.4 \times 10^{5} \mathrm{Tm}^{-1}$ in $x$ for $20 \mathrm{~mA}$ of current through the constriction. The $v$-encoding pulse is limited to $20 \mathrm{~mA}$ to avoid artifacts from the strong transverse field produced by the constriction during the gradient pulse ( $22 \mathrm{mT}$ at the tip of the sample for $20 \mathrm{~mA}$ ). In the future, such artifacts can be avoided by increasing $B_{0}$. Nonetheless, these gradients are more than $10^{4}$ times stronger than the highest gradients used in inductively detected MRI [38]. During the readout, the peak gradient is approximately $5.0 \times 10^{5} \mathrm{Tm}^{-1}$ for $71 \mathrm{~mA}$ (limited by our amplifiers) of current through the constriction, corresponding to a current density of $3.0 \times 10^{8} \mathrm{~A} \mathrm{~cm}^{-2}$.

While the primary aim of the present study is to demonstrate a new technique for nanoscale pulsed magnetic resonance, the spatial resolution achieved here is comparable to the best resolution obtained in nanoscale MRI [12]. Here, the resolution could be improved by working at higher static magnetic field strengths, which would enable stronger $v$-encoding pulses. In addition, the readout gradient could likely be increased by several times, which would potentially improve the SNR and resolution by a considerable amount. We have successfully tested several smaller constrictions that we have fabricated at current densities larger than $10^{9} \mathrm{~A} \mathrm{~cm}^{-2}$ without failure. We typically observe, however, that nanowires experience a small increase in force noise during operation of the constriction. The origin of this excess force noise is not totally clear but appears to be electrostatic in nature. To achieve the best SNR, this excess noise should be minimized to enable working at high current densities.

\section{SENSITIVITY}

When the measurement is dominated by detector noise, multiplexed techniques, such as Fourier-transform methods, greatly enhance sensitivity by allowing the simultaneous acquisition of multiple spectral components. In general, such methods improve sensitivity by a factor of $\sqrt{N}$ over sequential-point methods, where $N$ is the number of image points. This sensitivity boost is known as the multiplex advantage [6]. For inductively detected MRI of thermally polarized samples, the dominant noise source is voltage noise from the receiver circuitry, and Fourier encoding is commonly employed as an efficient method of imaging [39]. The multiplex advantage has also been exploited for force-detected NMR and MRI of thermally polarized samples [21-25], where force noise dominates.

If, however, the object of interest is statistically polarized, as is the case for nanometer-size samples, spin noise contributes to the total noise [40], and a new analysis is required. Below, we argue that for high-resolution imaging of statistically polarized samples, the detector noise effectively dominates the total noise for small voxel sizes. In this case, the multiplex advantage still holds, and Fouriertransform techniques offer a significant sensitivity boost for nanoscale MRI.

In the Supplemental Material [29], we show that in $d$ dimensions, the average SNR of an image acquired via Fourier encoding is

$\mathrm{SNR}=e^{-\tau_{p} / \tau_{m}}\left(\frac{2^{d} N \bar{A} \tau_{p}}{T}+\frac{2^{d} N S_{F}}{T \sigma_{\text {spin }}^{2}}+\frac{2^{d} N S_{F}^{2}}{4 T \tau_{p} \sigma_{\text {spin }}^{4}}\right)^{-1 / 2}$.

Here, $\sigma_{\text {spin }}^{2}$ is the variance of the spin component of the force signal from the entire sample, $S_{F}$ is the oscillator force-noise power spectral density, $T$ is the averaging time per point, and $\bar{A}$, which is approximately 2 for the present experiment, characterizes the average error in the autocorrelation integrated over the sample. Spin-relaxation effects have been neglected in the above estimate. For comparison, the average SNR of an image in which each voxel is measured sequentially is

$$
\mathrm{SNR}_{\mathrm{point}}=\left(\frac{2 \tau_{m}}{T}+\frac{2 S_{F}^{2} N^{2}}{T \tau_{m} \sigma_{\mathrm{spin}}^{4}}+\frac{2 S_{F} N}{T \sigma_{\mathrm{spin}}^{2}}\right)^{-1 / 2} .
$$

Equation (4) assumes that the signal in each voxel is the same (see the Supplemental Material [29]).

In both Eqs. (3) and (4), the first term in the parentheses represents the spin noise, the second term represents the oscillator force noise, and the last term is the covariance of the force noise and the spin noise. In Fourier encoding, the force-noise contribution scales more favorably with $N$ than in sequential-point imaging because all voxels in the image are measured $N$ times, compared with only once in the sequential-point case. This sensitivity enhancement exemplifies the multiplex advantage when detector noise dominates. The spin-noise contribution, however, scales less favorably with $N$ in Fourier encoding because spin noise from the entire sample contributes to every data point. 
When the number of image points is large enough, such that the force noise significantly exceeds the spin noise per voxel, i.e., when $N S_{F} / 2 \tau_{m} \sigma_{\text {spin }}^{2} \gg 1$, then $\mathrm{SNR}_{\text {point }} \propto$ $1 / N$, in contrast to Fourier encoding, where $\mathrm{SNR} \propto$ $1 / \sqrt{N}$. In this regime, the detector noise (i.e., force noise) effectively dominates, and Fourier encoding can be expected to offer better sensitivity. The present experiment, for example, has benefited from multiplexing because $\quad \sigma_{\text {spin }}^{2} \approx 300 \mathrm{aN}^{2}, \quad S_{F} \approx 10 \mathrm{aN}^{2} \mathrm{~Hz}^{-1}, \quad$ and $N S_{F} / 2 \tau_{m} \sigma_{\text {spin }}^{2} \approx 13$.

\section{CONCLUSION}

In this work, we have demonstrated nanoscale pulsed Fourier-transform magnetic resonance spectroscopy and imaging. Our technique relies on creating correlations in the spin noise of a nanoscale sample using rf and gradient pulses generated by a metal constriction. We have also argued that our technique provides a sensitivity enhancement for high-resolution nanoscale imaging via the multiplex advantage.

We conclude by noting several possible extensions of our work. First, our technique could be readily extended to enable full three-dimensional encoding with constrictions capable of producing two orthogonal static gradients. A small coil could also be used to generate a uniform rf field in the sample, which would enable the use of solid-state decoupling sequences, such as the magic sandwich [22]. These pulse sequences could be used for high-resolution spectroscopy and would permit longer encoding times and better spatial resolution in imaging. Such a coil, together with gradient pulses from the constriction, could also be employed to perform nanoscale tomography. More generally, our approach serves as a model for leveraging these and other sophisticated pulsed magnetic resonance tools to aid nanoscale MRI in its progress toward atomic-scale imaging.

\section{ACKNOWLEDGMENTS}

This work was supported by the Army Research Office through Grant No. W911NF-12-1-0341 and by the Department of Physics and the Frederick Seitz Materials Research Laboratory Central Facilities at the University of Illinois. Work at Northwestern University was supported by the National Science Foundation Grants No. DMI0507053 (E. R. H.) and No. DMR-1006069 (L. J. L.).

[1] Richard R. Ernst, Geoffrey Bodenhausen, and Alexander Wokaun, Principles of Nuclear Magnetic Resonance in One and Two Dimensions (Oxford University Press, New York, 1997).

[2] Magnetic Resonance Imaging, edited by David D. Stark and William G. Bradley (C. V. Mosby Co., St. Louis, 1988).
[3] S. Ogawa, T.-M. Lee, A. S. Nayak, and P. Glynn, Oxygenation-Sensitive Contrast in Magnetic Resonance Image of Rodent Brain at High Magnetic Fields, Magn. Reson. Med. 14, 68 (1990).

[4] D. Lebihan, E. Breton, D. Lallemand, P. Grenier, E. Cabanis, and M. Lavaljeantet, MR Imaging of Intravoxel Incoherent Motions-Application to Diffusion and Perfusion in Neurologic Disorders, Radiology 161, 401 (1986).

[5] R. R. Ernst and W. A. Anderson, Application of Fourier Transform Spectroscopy to Magnetic Resonance, Rev. Sci. Instrum. 37, 93 (1966).

[6] P. Fellgett, I. Les Principes Généraux des Méthodes Nouvelles en Spectroscopie Interférentielle-A Propos de la Théorie du Spectromètre Interférentiel Multiplex, J. Phys. Radium 19, 187 (1958).

[7] A. Kumar, D. Welti, and R. R. Ernst, NMR Fourier Zeugmatography, J. Magn. Reson. 18, 69 (1975).

[8] D. I. Hoult, Rotating Frame Zeugmatography, J. Magn. Reson. 33, 183 (1979).

[9] L. Bolinger and J.S. Leigh, Hadamard Spectroscopic Imaging (HSI) for Multivolume Localization, J. Magn. Reson. 80, 162 (1988).

[10] L. Ciobanu, D. A. Seeber, and C. H. Pennington, $3 D M R$ Microscopy with Resolution $3.7 \mu \mathrm{m}$ by $3.3 \mu \mathrm{m}$ by $3.3 \mu \mathrm{m}$, J. Magn. Reson. 158, 178 (2002).

[11] J. A. Sidles, J. L. Garbini, K. J. Bruland, D. Rugar, O. Zuger, S. Hoen, and C.S. Yannoni, Magnetic Resonance Force Microscopy, Rev. Mod. Phys. 67, 249 (1995).

[12] C. L. Degen, M. Poggio, H. J. Mamin, C. T. Rettner, and D. Rugar, Nanoscale Magnetic Resonance Imaging, Proc. Natl. Acad. Sci. U.S.A. 106, 1313 (2009).

[13] C. L. Degen, Scanning Magnetic Field Microscope with a Diamond Single-Spin Sensor, Appl. Phys. Lett. 92, 243111 (2008).

[14] J. M. Taylor, P. Cappellaro, L. Childress, L. Jiang, D. Budker, P. R. Hemmer, A. Yacoby, R. Walsworth, and M.D. Lukin, High-Sensitivity Diamond Magnetometer with Nanoscale Resolution, Nat. Phys. 4, 810 (2008).

[15] H. J. Mamin, M. Kim, M. H. Sherwood, C. T. Rettner, K. Ohno, D. D. Awschalom, and D. Rugar, Nanoscale Nuclear Magnetic Resonance with a Nitrogen-Vacancy Spin Sensor, Science 339, 557 (2013).

[16] T. Staudacher, F. Shi, S. Pezzagna, J. Meijer, J. Du, C. A. Meriles, F. Reinhard, and J. Wrachtrup, Nuclear Magnetic Resonance Spectroscopy on a (5-Nanometer $)^{3}$ Sample Volume, Science 339, 561 (2013).

[17] M.S. Grinolds, S. Hong, P. Maletinsky, L. Luan, M. D. Lukin, R. L. Walsworth, and A. Yacoby, Nanoscale Magnetic Imaging of a Single Electron Spin under Ambient Conditions, Nat. Phys. 9, 215 (2013).

[18] H. J. Mamin, R. Budakian, B. W. Chui, and D. Rugar, Detection and Manipulation of Statistical Polarization in Small Spin Ensembles, Phys. Rev. Lett. 91, 207604 (2003).

[19] H. J. Mamin, R. Budakian, B. W. Chui, and D. Rugar, Magnetic Resonance Force Microscopy of Nuclear Spins: Detection and Manipulation of Statistical Polarization, Phys. Rev. B 72, 024413 (2005).

[20] J. G. Kempf and J.A. Marohn, Nanoscale FourierTransform Imaging with Magnetic Resonance Force Microscopy, Phys. Rev. Lett. 90, 087601 (2003). 
[21] C. L. Degen, Q. Lin, A. Hunkeler, U. Meier, M. Tomaselli, and B. H. Meier, Microscale Localized Spectroscopy with a Magnetic Resonance Force Microscope, Phys. Rev. Lett. 94, 207601 (2005).

[22] C. L. Degen, Q. Lin, and B. H. Meier, Dipolar Spin Echoes in Magnetic Resonance Force Microscopy, Phys. Rev. B 74, 104414 (2006).

[23] K.W. Eberhardt, C. L. Degen, and B.H. Meier, Fast Magnetic Resonance Force Microscopy with Hadamard Encoding, Phys. Rev. B 76, 180405(R) (2007).

[24] K. W. Eberhardt, A. Hunkeler, U. Meier, J. Tharian, S. Mouaziz, G. Boero, J. Brugger, and B.H. Meier, TwoDimensional Magnetic Resonance Force Microscopy Using Full-Volume Fourier and Hadamard Encoding, Phys. Rev. B 78, 214401 (2008).

[25] R. Joss, I. T. Tomka, K. W. Eberhardt, J. D. van Beek, and B. H. Meier, Chemical-Shift Imaging in Micro- and NanoMRI, Phys. Rev. B 84, 104435 (2011).

[26] J. M. Nichol, E. R. Hemesath, L. J. Lauhon, and R. Budakian, Displacement Detection of Silicon Nanowires by Polarization-Enhanced Fiber-Optic Interferometry, Appl. Phys. Lett. 93, 193110 (2008).

[27] D. E. Perea, E. Wijaya, J. L. Lensch-Falk, E. R. Hemesath, and L.J. Lauhon, Tomographic Analysis of Dilute Impurities in Semiconductor Nanostructures, J. Solid State Chem. 181, 1642 (2008).

[28] J. M. Nichol, E. R. Hemesath, L. J. Lauhon, and R. Budakian, Nanomechanical Detection of Nuclear Magnetic Resonance Using a Silicon Nanowire Oscillator, Phys. Rev. B 85, 054414 (2012).

[29] See Supplemental Material at http://link.aps.org/ supplemental/10.1103/PhysRevX.3.031016 for details on the fabrication of the constriction, time-averaged autocorrelation function, image reconstruction, and signal-to-noise ratio.

[30] N.F. Ramsey, A Molecular Beam Resonance Method with Separated Oscillating Fields, Phys. Rev. 78, 695 (1950).

[31] M. Garwood and L. DelaBarre, The Return of the Frequency Sweep: Designing Adiabatic Pulses for Contemporary NMR, J. Magn. Reson. 153, 155 (2001).

[32] Paul J. Carson, Louis A. Madsen, Garett M. Leskowitz, and Daniel P. Weitekamp, U.S. Patents No. 6,078,872 and No. 6,081,119 (2000).

[33] Garett M. Leskowitz, Ph.D. dissertation, California Institute of Technology, 2003.

[34] M. Poggio, C. L. Degen, C. T. Rettner, H. J. Mamin, and D. Rugar, Nuclear Magnetic Resonance Force Microscopy with a Microwire rf Source, Appl. Phys. Lett. 90, 263111 (2007).

[35] M.F. Froix, D. J. Williams, and A. O. Goedde, NMR Relaxation Time Studies of Polystyrene, Macromolecules 9, 354 (1976).

[36] C.P. Slichter, Principles of Magnetic Resonance (Springer, New York, 1990).

[37] $E_{u}\left(t_{u}\right)$ could not be measured in the present experiment because the constriction produces a highly inhomogeneous rf field.

[38] S.-C. Lee, K. Kim, J. Kim, S. Lee, J.H. Yi, S. W. Kim, K.-S. Ha, and C. Cheong, One Micrometer Resolution NMR Microscopy, J. Magn. Reson. 150, 207 (2001).

[39] P. Brunner and R. R. Ernst, Sensitivity and Performance Time in NMR Imaging, J. Magn. Reson. 33, 83 (1979).

[40] C. L. Degen, M. Poggio, H.J. Mamin, and D. Rugar, Role of Spin Noise in the Detection of Nanoscale Ensembles of Nuclear Spins, Phys. Rev. Lett. 99, 250601 (2007). 